\title{
REFLEXÕES SOBRE A POLÍTICA PÚBLICA NA CIDADE: AS CONQUISTAS E OS DESAFIOS DA SAÚDE MENTAL DE QUIXADÁ
}

\author{
Thiago Sousa Felix \\ Universidade Federal do Ceará - UFC \\ Aluísio Ferreira de Lima \\ Universidade Federal do Ceará - UFC
}

\begin{abstract}
Resumo
Passados quase um quarto de século desde a criação do Centro de Atenção Psicossocial de Quixadá, Ceará, chegamos a um momento no qual as análises e avaliações são bem-vindas e mesmo indispensáveis. O presente relato de experiência objetiva refletir alguns aspectos referentes ao processo de formação da política de saúde mental de Quixadá. Consideramos as particularidades das experiências de saúde nessa cidade que se evidenciam através de algumas pesquisas de pós-graduação que vem sendo realizadas. Faz parte ainda do texto uma série de proposições que se colocam como possibilidades para futuros aprimoramentos dessa política municipal.

Palavras-chave: saúde mental; políticas públicas; Reforma Psiquiátrica.
\end{abstract}

\section{REFLECTIONS ON PUBLIC POLICY OF THE CITY: AS ACHIEVEMENTS AND CHALLENGES OF MENTAL HEALTH OF QUIXADÁ}

\begin{abstract}
After almost a quarter of a century since the creation of the Psychosocial Care Center of Quixadá city, Ceará, we come to a time when analysis and evaluations are welcomed and even needful. This report aims to think over about some aspects of the process of formation of the mental health policy in Quixadá. We consider the particularities of this healthcare experience, evident through of some postgraduate researches. Composing the text there are several propositions that arise as possibilities for future improvements within this municipal policy.

Keywords: mental health; public policy; Psychiatric reform.
\end{abstract}

\section{REFLEXIONES SOBRE POLÍTICAS PÚBLICAS DE LA CIUDAD: LOS LOGROS Y RETOS DE LA SALUD MENTAL DE QUIXADÁ}

\begin{abstract}
Resumen
Después de casi un cuarto de siglo desde la creación del Centro de Atención Psicosocial de Quixadá, Ceará, llegamos a un momento en que los análisis y las evaluaciones son recibidos e incluso indispensable. Este informe tiene como objetivo reflejar la experiencia de algunos aspectos del proceso de formación de la política de salud mental de Quixadá. Consideramos que las características particulares de la experiencia de atención médica en esta ciudad que son evidentes a través de varias investigaciones de posgrado. Aún así parte del texto una serie de proposiciones que surgen como posibilidades para futuras mejoras de esta política municipal.

Palabras clave: salud mental; políticas públicas; Reforma Psiquiátrica.
\end{abstract}

\section{INTRODUÇÃO}

O processo de redemocratização do Brasil no final dos anos 1980 permitiu aos municípios ter uma maior abertura política para discutir as questões das cidades. Vale destacar que até o completo processo da referida redemocratização, os prefeitos das capitais e governadores dos Estados eram 
indicados pelo governo federal para os chamados cargos biônicos, ou seja, sem o sufrágio das urnas.

O clima de abertura política influenciou as várias esferas da vida dos brasileiros no sentido de uma maior descentralização administrativa e de maior participação política. Tratando da saúde mental, Amarante (1992) assevera que o planejamento em saúde mental teria como cenário as microrregiões e não mais os poderes centrais distantes das pessoas.

Com uma relativa independência financeira acrescida de uma mais ampla independência de gestão, o resultado na prática foi um estímulo ao surgimento de soluções locais (nos níveis municipais e estaduais), que significou a antecipação de políticas que se tornaram nacionais como é o caso do Programa dos Agentes Comunitários de Saúde, criado no Ceará em 1987 (Barros, Barbiere, Ivo \& Silva, 2010). Como também no campo da saúde mental, por exemplo, em cidades como Iguatu, Sobral e Quixadá com a criação de Centros de Atenção Psicossocial. Esta última constitui-se no tema do presente trabalho.

Esse processo histórico não foi resultado do acaso, nem de uma única "cabeça pensante". De acordo com o levantamento de Bleicher (2015), ocorreu a aglutinação de jovens lideranças oriundas de movimentos sociais progressistas da política, da universidade e da militância na saúde que inclusive haviam participado ativamente da criação do Sistema Único de Saúde (SUS) nos âmbitos nacional e estadual. Esse encontro de vários atores sociais foi designado de "confluência de astros" (Bleicher, 2015). Deve-se notar que o movimento tal como ocorreu em Quixadá foi distinto do que adveio na maioria das cidades do Ceará.

Em outras cidades do Estado, os gestores contavam quase que exclusivamente com recursos humanos locais para operacionalizar as políticas que eram implementadas no âmbito federal. Quixadá (que juntamente com as cidades de Iguatu, Canindé, Cascavel, Aracati compôs o pioneirismo na inauguração dos CAPS no interior do estado, entre os anos de 1992 e 1996), ao contrário, contou com a vinda de dezenas de técnicos de várias regiões do Brasil que buscaram criar na cidade um modelo de saúde pública mais pela militância política do que pelo interesse profissional individual ou financeiro. Esse modelo visava ainda romper com tudo que representasse o velho jeito de fazer política no interior do Ceará. Assim, como iniciativa local e não como proposição do Ministério da Saúde, a criação da assistência à saúde mental de Quixadá ocorrida em 13 de dezembro de 1993, representou mais que um ganho de serviço acessível à população: significou a possibilidade de experiências exitosas partindo da esfera municipal.

Avaliando o processo de implantação dos Centros de Atenção Psicossocial (CAPS) no Ceará, Sampaio \& Santos (2016) consideram esse processo como, propriamente, uma vitória da municipalização e da interiorização dos serviços de saúde. Certamente os autores identificam as várias nuances desse processo e 
conseguem fazer a crítica de toda a burocracia e as limitações inerentes ao sistema indo muito além do financiamento que é posto usualmente como a queixa corriqueira nos debates sobre o tema. Segundo os mesmos, Quixadá foi pioneira, no âmbito estadual, na "inclusão de supervisão institucional sistemática, no desenvolvimento de uma política de formação de trabalhadores especialmente para a Reforma Psiquiátrica, no atendimento domiciliar de emergência". (2016, p. 166).

Enquanto alguns setores contribuíam para Fortaleza, a capital do estado, permanecer à margem das inovações da Reforma Psiquiátrica que já transcorria em outros lugares do país, iniciava-se em algumas cidades do interior processos que seriam um importante componente na implementação das políticas de saúde (Sampaio \& Santos, 2016). O movimento de transformação da rede de atenção psicossocial no Ceará, portanto, começou da iniciativa de gestões municipais no interior e não de empreendimentos da prefeitura ou do governo estadual na capital o que só vieram a acontecer efetivamente depois da Lei no 12.151 de 1993, conhecida como Lei Mário Mamede.

No caso de Quixadá, em conformidade com a agenda que efetivava a participação popular nas questões municipais, ocorreu a criação das comissões municipais de saúde e, posteriormente, a realização das conferências de saúde também baseadas na Lei no $8.142 / 90$ que regula a participação comunitária no contexto da saúde. Pode-se afirmar que a realização da Conferência de Saúde e várias conferências nos distritos sede e na zona rural do município geravam a possibilidade do cidadão opinar sobre as políticas legitimando pautas (como da Reforma Psiquiátrica), criando demandas e se apropriando da gestão no âmbito local.

A citação a seguir ilustra o significado das experimentações de Quixadá no campo da atenção básica à saúde e suas repercussões nas formulações de políticas públicas para o Brasil no contexto da criação do Programa da Saúde da Família (PSF):

As ideias pensadas e formuladas no âmbito do Ministério da Saúde de Santilo nos idos de 1993 tinham, segundo Girade, forte influência de algumas experiências em andamento no País, como as de Niterói, do Grupo Hospitalar Conceição, ligado ao Ministério da Saúde, da SES de São Paulo e de Quixadá, no Ceará (...). O fato é que, no dia 27 de dezembro de 1993, se reuniram, no Ministério da Saúde, representantes das experiências citadas, alguns consultores, e, então, foram definidas as bases do que viria a ser o Programa Saúde da Família (...). (Andrade \& Barreto, 2010, p. 95).

Diga-se que a implantação do PSF e a estruturação do SUS, deu-se numa conjuntura inicial amplamente desfavorável tendo em vista a instabilidade política do período com o impeachment do Presidente Fernando Collor de Mello em 1992. Naquele mesmo ano, ocorria a IX Conferência Nacional de Saúde que trazia como tema: "Saúde: Municipalização é o Caminho". 
As Conferências Municipais em Quixadá, além de outras assembleias e reuniões populares que ocorriam nas praças, escolas e auditórios faziam parte das estratégias de municipalização dos serviços de saúde dentre outras políticas (Bleicher, Freire \& Sampaio, 2014, p. 531) e eram mesmo uma marca da gestão na cidade. Esse modo de gerir buscando estimular a participação popular, numa região onde o comando esteve centralizado na figura patriarcal dos coronéis, foi de grande impacto na cultura local e dividiu as opiniões dos cidadãos.

As ações de maior impacto na saúde local tiveram início na primeira gestão de Ilário Marques (1992-1996). Como já referido anteriormente, as iniciativas daquele período contaram com a participação de profissionais militantes que ansiavam pela construção de um sistema público de assistência à população. Como atestam os diversos relatos colhidos na pesquisa de Bleicher (2015), a derrota nas eleições municipais de 1996 do grupo político que atuou na construção dos serviços de saúde no município e a quase expulsão dos profissionais de saúde que foram apelidados jocosamente de forasteiros, gerou uma desarticulação dos serviços e representou quase o desfecho precoce daquelas experiências pioneiras em saúde. Mesmo com formação médica, a vitória eleitoral de Francisco Mesquita representava, naquele momento, a derrota do projeto político do antecessor, causando, assim, certa desarticulação na rede de assistência à saúde.

Apesar de não serem retomadas com o mesmo vigor, as ações que almejavam prosseguir o processo de municipalização dos serviços de saúde ocorreram somente na gestão (2000-2004) e ainda na seguinte (2004-2008) quando Ilário Marques se reelegeu pela terceira vez. O fato é que desde então tem sido um desafio constante preencher as vagas de profissionais de saúde completando as equipes de saúde da família, notadamente nas localidades mais distantes da sede do município.

Essa gestão significou a retomada de uma nova agenda, porém, sem o "brilho" e a presença de muitos dos profissionais militantes que haviam participado da implantação da política de saúde municipal (Bleicher, 2015). No pleito municipal de 2008, foi eleito o médico Rômulo Carneiro, candidato da situação, que, até certo ponto deu continuidade ao grupo político prosseguindo com o projeto dos anos anteriores. O rompimento político entre prefeito e seu antecessor só ocorreu meses antes das eleições municipais de 2012.

Marcou novamente uma ruptura política no município o pleito de 2012 no qual foi eleito o empresário João da Sapataria (algo semelhante ao que ocorreu em 1996, com a eleição do prefeito Francisco Mesquita). Apesar dos esforços iniciais, poucas iniciativas locais se colocaram como originais em termos de transformação na gestão e assistência à saúde tal como ocorreu nos primeiros anos da década de 1990. Contudo, os novos tempos trouxeram a possibilidade de realizar parcerias, aproveitando o conceito do qual a cidade dispunha. 
Um fenômeno determinante para o campo da saúde na cidade foi o surgimento de cursos de graduação da área da saúde com a fundação da Unicatólica ligada à Diocese de Quixadá (é uma instituição de ensino superior filantrópica que agrega cursos do campo da saúde, dentre outras áreas), em 2004. Passados os primeiros anos desde sua criação, a Unicatólica se tornou parceira da prefeitura em várias ações no município, além de prestar assistência através dos estágios e projetos de extensão.

Deve-se fazer menção, também, ao Programa de Educação pelo Trabalho para a Saúde - PET Saúde que foi uma parceria realizada entre a Unicatólica e a Prefeitura Municipal. No projeto iniciado pela Instituição de Ensino Superior (IES) em 2013, o tema central a ser articulado na rede municipal foi o da saúde mental. Assim, os estudantes da Unicatólica atuaram em espaços de saúde da cidade tendo como preceptores os profissionais dos CAPS e da Estratégia de Saúde da Família (ESF) que se reuniam para o planejamento das atividades. Em 2013, temos notícia também da implantação da Residência Integrada em Saúde (RIS), uma residência multiprofissional que foi instalada em Fortaleza e em outras nove cidades escolhidas no interior do estado, dentre elas Quixadá. Esse projeto do Governo do Estado estava sob a tutoria da Escola de Saúde Pública do Ceará.

\section{MÉTODO}

Além dos trabalhos de conclusão de curso e relatórios fomentados por estudantes e pesquisadores de várias instituições de ensino, inclusive aquelas instaladas na cidade, como a Unicatólica, a discussão sobre a rede de assistência à saúde, e especificamente, de saúde mental de Quixadá foi tema de diversas pesquisas (que devem ser melhor sistematizadas em trabalhos com esta finalidade específica). A bibliografia sobre a rede de saúde na cidade é tão variada quanto a obra organizada por Carneiro, Ruiz, Landim e Sampaio (2006) fruto de uma parceria entre a Universidade Estadual do Ceará e a prefeitura ou o estudo de Fuhrer (2010), este último originário dos Estados Unidos, intitulado "Tem um Doido no CAPS / Que diz que Louco é Gente/Que diz que Louco Sabe/Que diz que Louco Sente".

Diante dessa diversidade de trabalhos, selecionamos aqueles que estivessem mais relacionados com o tema central desse estudo. Dessa forma, os que se farão presentes em referências e citações nesse relato de experiência, serão, mais especificamente, os trabalhos de Sampaio e Santos (2016), Bleicher et al. (2014), Bleicher (2015) e Oliveira, Guimarães, Carneiro e Sampaio (2014) por considerarmos esses como indispensáveis para entender as conquistas e limites da Saúde Mental de Quixadá.

Essas referências bibliográficas serão articuladas com as percepções do primeiro autor desse relato que trabalha já fazem muitos anos nos serviços 
substitutivos da cidade. Acreditamos que isso permitirá apresentar reflexões críticas e algumas considerações acerca de caminhos possíveis para o aperfeiçoamento dessa política pública.

\section{AS EQUIPES E A CONSTRUÇÃo DE UMA POLÍtICA DE SAÚde MENTAL EM QUIXADÁ}

Quase vinte anos depois da implantação do Centro de Atenção Psicossocial - CAPS Geral em Quixadá veio a criação do Centro de Atenção Psicossocial Álcool e Outras Drogas - CAPS AD, em 2011, denominado Casa Norte. Os dois serviços de saúde mental especializados da cidade compartilham vínculo que tem favorecido, apesar de algumas rupturas, caminharem juntos depois da mudança do CAPS AD para sua sede. Apesar de serem dois serviços com estruturas e públicos específicos distintos, as equipes permaneceram integradas de tal forma que estiveram acompanhadas pela mesma supervisão e ainda hoje trocam a indispensável colaboração mútua para resoluções dos casos clínicos, questões burocráticas e realização de ações conjuntas.

Podemos afirmar com Ferioti (2016) que as equipes de saúde mental da cidade se constituem num "coletivo não competitivo, que se constitui de maneira oposta à lógica neoliberal e aos modos fragmentados de organização do trabalho e produção de saber". (Ferioti, 2016, p. 27). As referidas equipes podem se ufanar por manterem pequenas/grandes formulações no campo estratificado da assistência à saúde onde paira o corporativismo e a burocracia tanto engessada como reprodutivista (que apenas reproduz práticas sem questioná-las ou problematizá-las na sua eficácia e coerência com a realidade quixadaense). Bleicher destaca algumas dessas iniciativas no trecho a seguir:

Com apenas um ano de criação, foi realizado um primeiro esforço de sistematização de sua experiência e propostas para o campo, por meio do Manual de Organização do Centro de Atenção Psicossocial de Quixadá, publicado originalmente na forma de apostila pela Secretaria de Saúde de Quixadá, posteriormente disponibilizado em formato eletrônico na internet. (Bleicher, 2015, p. 19).

No CAPS de Quixadá, criou-se também a função do auxiliar de saúde mental, um cargo específico que integra os mais variados trabalhadores do serviço: do motorista ao cozinheiro, do recepcionista ao zelador. Essa, na visão da equipe, foi uma maneira de prestigiar os profissionais de nível médio e fundamental que não tinham formação acadêmica e que são de fundamental importância numa assistência de qualidade ao usuário do SUS. Apesar de não possuir maiores benefícios como remuneração, a denominação possibilitou criar uma identidade em torno do serviço e do tema saúde mental.

Empreendimentos dessa envergadura, fortalecidas pelas supervisões e reuniões de equipe, também propiciaram certa coesão dos trabalhadores em torno de um "projeto de Reforma Psiquiátrica". Em outras palavras, permitiram 
uma ação direcionada para transformação do cotidiano das pessoas que buscavam o serviço, pois questionadora do estigma da loucura mais do que numa perspectiva teórica: numa ação prática. As atitudes dos profissionais da saúde mental na cidade visavam, de maneira difusa, equacionar o estigma em relação à loucura, dando cidadania ao portador de sofrimento psíquico.

\begin{abstract}
A ideia de que as pessoas circulassem pela cidade era uma das principais estratégias para o combate ao estigma e para uma possibilidade de cidadania do psicótico. Wellington Peixoto de Sousa, que iniciou sua trajetória profissional no CAPS como auxiliar de Enfermagem e, posteriormente, se tornou técnico de Enfermagem e coordenador do serviço, relata que, ainda antes do CAPS ser inaugurado, foram realizadas supervisões para que os trabalhadores tivessem uma ideia de como iriam atuar - e, para ele, o principal da atuação do CAPS era acabar com o estigma (SOUSA, 2013, p. 13). Enquanto isso, para Carlos Magno Cordeiro Barroso, seu contemporâneo médico e, posteriormente, psiquiatra e supervisor do serviço, definiu como a principal questão do serviço a cidadania do psicótico. (Bleicher et al., 2014, p. 534).
\end{abstract}

O protagonismo das equipes do CAPS Geral e CAPS AD de Quixadá no processo de constituição dos serviços a serem disponibilizados para a população ocorreu de modo semelhante ao de outras experiências pioneiras na área no tocante à realização de atividades que estavam além do script e principalmente da Autorização de Procedimentos de Alta Complexidade (APAC), mecanismo operacionalizado no SUS que permitia o pagamento pelo atendimento realizado no serviço (Ministério da Saúde, 2002). Essas atividades eram: mobilizações, intervenções culturais, reuniões temáticas, produção de materiais, de pesquisas locais, atendimentos infantis, grupais e uma variada gama de ações que visavam subsidiar a gestão municipal na resolução de demandas concernentes à saúde no município.

Mantendo tal prática da realização de atividades que ocorrem nos espaços da cidade e, portanto, fora dos muros do CAPS (mantendo o entusiasmo que embalou os primeiros anos) prosseguem ainda as experiências de caráter comunitário que visam potencializar a mobilização política e soluções solidárias que envolvem a participação social como é o caso da Universidade Popular (coordenada pelo CAPS Geral) e do Laboratório Social (dinamizada pelo CAPS AD) (Oliveira et al., 2014).

A cidade se encontrava articulada numa dinâmica que os autores Sampaio e Santos (2016) denominaram como "sistema de ação pedagógica" que integrava: Seminários de aprofundamento teórico, reunião semanal de estudo, discussão de casos, realização de pesquisas, supervisão institucional, jornadas científico-culturais de curta duração, formação técnico política com outros sujeitos da rede, participação em eventos científicos, dentre outros.

Exemplo significativo desse movimento criativo e de resistência está na realização da Jornada de Saúde Mental e Cidadania de Quixadá que se constitui num espaço de discussão, de aprofundamento permanente das questões da 
saúde pública e ainda propicia um momento de integração entre profissionais e militantes de saúde de todo o Ceará. A Jornada ocorre ininterruptamente desde o ano de 1992 (portanto antes da criação do próprio CAPS) se configurando como um capítulo da história da saúde mental no Ceará e no Brasil a ser estudado num espaço mais oportuno.

Parte do êxito de participação nas jornadas municipais se dá justamente porque ela aglutina as reflexões sobre a Reforma Psiquiátrica e questões relacionadas à saúde pública num espaço alternativo aos círculos que existem na capital do estado. Dessa forma, a Jornada se configurou, com o tempo, num espaço para quem ansiava por refletir as demandas da rede de atenção psicossocial. É possível que o movimento para um debate mais especializado no campo da saúde mental tenha atraído profissionais e estudiosos de fora, enquanto usuários do sistema municipal foram se afastando.

Sabemos, contudo, que apesar da boa intenção dos profissionais e de todas as portarias ministeriais que fundamentam uma assistência humanizada que favoreça a autonomia e a cidadania dos usuários do SUS, muitos conceitos e intenções se perdem na burocracia, nas carências estruturais da rede, nas carências socioeconômicas da população, nas demandas vivas dos portadores de sofrimento psíquico.

Além da própria equipe que acolhe estudiosos que investigam a atenção psicossocial e de profissionais interessados em conhecer mais sobre saúde mental, há registros de informações sobre os eventos como a Jornada, com vídeos, panfletos e outros materiais, as atas das reuniões de equipe, documentações e literatura especializada que compõem o acervo do CAPS Geral. Esses registros e materiais têm permanecido à disposição para a consulta de pesquisadores e estudantes e servido de fonte de pesquisa para dissertações de mestrado como o de Páscoa (2008), doutorado como o de Bleicher (2015), além do trabalho de Fuhrer (2010).

Os arquivos possibilitam compreender as mudanças que ocorreram criando um quadro de antes e depois da criação do serviço:

Antes da implantação do CAPS, a Secretaria de Saúde encaminhava para Fortaleza uma média de duas internações psiquiátricas/mês. Após a implantação, o encaminhamento reduziu-se para algo em torno de uma internação a cada oito meses, pois a equipe do CAPS tem podido conter situações agudas nas residências, através de visita domiciliar e em leitos do Hospital Geral. O pessoal do Hospital tem aprendido a conviver com as exigências diferenciadas de um cliente psicótico em crise e ajudando a garantir a permanência média de sete dias por internação. (Sampaio \& Santos, 2016, p. 171).

Os autores finalizam com uma nota otimista trazendo esperança e inspiração para a militância que continuamente atua na saúde: "O modelo extrahospitalar, alternativo, crítico-social, democrático, de assistência à saúde mental, ética e politicamente competente, também está demonstrando ser tecnicamente competente." (Sampaio \& Santos, 2016, p. 172). 
Chegamos, sem embargo, num tempo em que a saúde mental, por mais avançada e competente que esteja, se torna invasiva, catalogando mais que diagnósticos, mas também histórias de vida e sofrimento. A indiferença com que tudo isso é tratado, longe de gerar cura, pode gerar ainda mais sofrimento psíquico.

O avanço da cultura da medicalização, por outro lado, que se faz presente nos três níveis de atenção à saúde, vem alarmando especialistas e a gestão municipal. Essa questão é uma ínfima amostra de um sistema que, empenhado em administrar a dor física e o sofrimento psíquico, esqueceu-se de sua potência criativa, das diversas maneiras de lidar com a realidade. Urge a possibilidade de uma abordagem que supere uma lógica consumista focada no prazer individual generalizado, inclusive, nos serviços de saúde do país onde a assistência à saúde se tornou sinônimo de medicalização da vida.

Retornando para as questões locais numa perspectiva propositiva e prognóstica, devemos elencar, em tópicos acrescidos de breves comentários, alguns dos pontos que consideramos pertinentes para o presente e o futuro da saúde mental de Quixadá, tais como: 1 - Promoção do resgate da participação popular nas decisões da política de saúde mental; 2 - Enfrentamento dos desafios da Residência Terapêutica; 3 - Reflexão dos serviços que irão compor a Rede de Atenção Psicossocial (RAPS) como a Unidade de Acolhimento (UA), por exemplo; 4 - Incremento do empreendedorismo solidário e o potencial artístico local; 5 - Criação de um Observatório e/ou Centro de Estudos em Saúde Mental; 6 - Implantação imediata do Plano de Cargos e Carreiras da Saúde.

Apesar do afastamento popular identificado por Bleicher (2015) em relação à experiências ocorridas em gestões municipais anteriores, é possível fomentar um espírito democrático e participativo a partir do cotidiano da saúde mental. Mesmo diferente do que se vivenciou em outros períodos históricos, é possível articular espaços como o Laboratório Social, a Universidade Popular e outras iniciativas comunitárias, se bem articulada com as lideranças locais, pactuada entre as secretarias municipais e a rede.

Reconhecida demanda local, as Residências Terapêuticas, presentes em várias experiências no país e mundo afora, seriam mais uma alternativa de moradia para pacientes cronificados que vivem sozinhos ou sofrem abandono familiar. A iniciativa ocorreria em consonância com os demais progressos que a cidade vivenciou no campo da saúde.

Por representar, de alguma forma, o estigma da internação, a Unidade de Acolhimento Infanto-Juvenil (UA) em processo de construção (interrompido por tempo indeterminado) na cidade, deve ser analisada com certa desconfiança. Esse serviço, ligado à pasta da saúde, quase inexistente em outras cidades do estado, pode apresentar várias limitações na sua operacionalização (inclusive, indo na contramão dos princípios da reforma psiquiátrica antimanicomial) e, paradoxalmente, será a única referência oferecida pelo poder público para a 
proteção de crianças e adolescentes que se encontram com necessidade de acolhimento transitório em saúde mental.

A cidade possui variadas experiências exitosas no campo do associativismo e da produção artística se configurando como polo cultural do cinema e das artes plásticas. Muitos artesãos, inclusive, mantém variada produção de souvenires que abastecem o comércio do turismo local. Apesar, contudo, de todo esse potencial, inexiste eventos temáticos e muito menos um trabalho permanente que envolva sujeitos com sofrimento psíquico.

A ideia do Centro de Estudos não é original, tendo em vista já ser vislumbrada, de diferentes formas, por profissionais e pesquisadores como Jackson Sampaio, Francimeiry Amorim, dentre outros. Invariavelmente, tal iniciativa requer comprometimento de agentes mais influentes como é o caso do Governo do Estado ou mesmo do Governo Federal.

Já o Plano de Cargos e Carreiras, é essencial para materializar o reconhecimento aos profissionais que atuam no campo da saúde. Somada à iniciativa anterior, tem o potencial de atrair e fixar profissionais com qualificação tanto para intervenção, como para o ensino e a pesquisa em saúde mental.

\section{CONSIDERAÇÕES FINAIS}

A implementação de uma política pública não se resume a publicações de leis, portarias e decretos (ou ao simples cumprimento das mesmas). Sem diretrizes operacionais eficazes, planejamento local, reflexão crítica e capacitação dos agentes responsáveis pela política o conteúdo dos papéis não se transferem para a realidade dos serviços. Também não surge de ideais e sujeitos isolados, mas da junção de vários fatores.

Despojar-se da pretensa superioridade cientificista, buscar soluções reais com pacientes e familiares, encaminhar na rede as demandas do usuário, não além dele, mas junto com ele. Num movimento coerente com as bandeiras da Reforma Psiquiátrica que clamam por cidadania e envolvimento social do sujeito com sofrimento psíquico.

Quixadá parece ter descoberto uma das principais estratégias para a gestão do cuidado em saúde mental: a construção de uma cultura antimanicomial no cotidiano da cidade. Partindo da liberdade gerencial que desfrutamos nas cidades nas últimas democráticas décadas, a realidade manicomial passou a ser apenas uma decisão política e não mais uma barreira intransponível e insuperável.

A situação estrutural representa bem a condição de subfinanciamento a que vem sendo legado o SUS de modo geral e a saúde mental de modo específico. Se em Quixadá, a identificação com os ideais da Reforma Psiquiátrica e com sua prática de emancipação estivesse associada às condições físicas da rede (prédios, recursos de trabalho, salários...), as equipes permaneceriam 
deveras frustradas. Se muito daquele ímpeto inicial se perdeu, restou ainda a militância que, aproveitada oportunistamente pelas gestões municipais e reconhecidas de forma generosa pela população, vai sendo transmitida pelos profissionais mais antigos e absorvidas pelos profissionais que ingressam no serviço.

Devem-se ponderar, não obstante, os limites e o impacto da vida política local na execução das políticas municipais de assistência. Restam os permanentes desafios políticos, tal como as gestões que se sucedem com prioridades distintas, que impactam de muitas formas o cotidiano dos serviços; os desafios de gestão, de formação, de assistência da população. Percebe-se, por exemplo, o desafio de articular soluções locais com problemáticas que, generalizadas mundialmente, definem-se não somente como questões de grupos humanos, mas da humanidade.

Essa série de pioneirismos torna-se, por fim, um agente desafiador que requer constante autocrítica, busca por superação e resultados originais que, além de receber normatizações das políticas federais e instruções ministeriais, contribui com novas perspectivas para essas políticas. Aliás, não há desafio maior do que sempre superar os seus próprios limites.

Nessas reflexões, a despeito de toda uma série de crises: da política até as econômicas, dos problemas estruturais do SUS, entender a importância do diálogo, do relato e do registro das experiências relacionadas à saúde mental como ato militante, político, além de científico. Enquanto prática, realizar autocrítica técnica e ética. Enquanto política, não desistir da utopia.

\section{DECLARAÇÃO DE CONFLITOS DE INTERESSE}

Não há conflitos de interesse.

\section{REFERÊNCIAS}

Amarante, P. D. C. (1992). A trajetória do pensamento crítico em saúde mental no Brasil: Planejamento na desconstrução do aparato manicomial. In $\mathrm{M}$. Kalil (Ed.), Saúde Mental e cidadania no contexto dos sistemas locais de saúde (pp. 103-119). São Paulo, Salvador: Editora Hucitec, Cooperação Italiana em Saúde.

Andrade, L. O. M., \& Barreto, I. C. H. C. (2010). Da reflexão crítica no movimento estudantil à participação na construção da estratégia saúde da família. In Ministério da Saúde, Secretaria de Atenção à Saúde, Departamento de Atenção Básica (Ed.), Memórias da saúde da família no Brasil (pp. 52-56). Brasília, DF: Ministério da Saúde. Recuperado de http://189.28.128.100/dab/docs/publicacoes/geral/livro_15.pdf 
Barros, D. F., Barbiere, A. R., Ivo, M. L., \& Silva, M. G. (2010). O contexto da formação dos agentes comunitários de saúde. Texto Contexto Enfermagem, 19(1), 78-84. doi:10.1590/S0104-07072010000100009

Bleicher, T. (2015). A política de Saúde Mental de Quixadá, Ceará (1993-2012): uma perspectiva histórica do sistema local de Saúde (Tese de doutorado). Universidade Estadual do Ceará, Universidade Federal do Ceará e Universidade de Fortaleza, Fortaleza, Ceará.

Bleicher, T., Freire, J. C., \& Sampaio, J. J. C. (2014). Avaliação de política em saúde mental sob o viés da alteridade radical. Physis, 24(2), 527-543. doi: $10.1590 /$ S0103-73312014000200011

Ministério da Saúde. Secretaria de Assistência à Saúde. (2002). Portaria n. 189, de 22 de Março de 2002 - complementa a Portaria GM/MS No 336, de 19 de fevereiro de 2002. Brasília, DF: autor. Recuperado de http://www.mp.go.gov.br/portalweb/hp/2/docs/189.pdf

Carneiro, C., Ruiz, E., Landim, L., \& Sampaio, J. (2006). Acolher cidadão: Estratégia de aperfeiçoamento do SUS em Quixadá, Ceará. Fortaleza, CE: Lhuas-Uece.

Ferioti, M. L. (2016). Patrimônio da reforma psiquiátrica: Sobre coletivos e incertezas. In J. A. Fernandes \& G. W. S. Campos (Eds.), Reconhecer o patrimônio da Reforma Psiquiátrica: O que queremos reformar hoje? (pp. 27-32). São Paulo, SP: Hucitec.

Fuhrer, Z. (2010). "Tem um Doido no CAPS / Que diz que Louco é Gente / Que diz que Louco Sabe / Que diz que Louco Sente". Failures and Successes in Brazilian Mental Health Reform: A Case Study of CAPS - Quixadá [Monografia]. Recuperado http://digitalcollections.sit.edu/isp_collection/857

Oliveira, M. A., Guimarães, A., Carneiro, C., \& Sampaio, J. J. C. (2014). Sofrimento psíquico e a cultura contemporânea: Perspectivas teóricoclínicas. Fortaleza, CE: EdUece.

Páscoa, J. G. O. (2008). Centro de Atenção Psicossocial de Quixadá: contribuições para mudanças na condução da política saúde mental no nível municipal (Dissertação de mestrado). Universidade Estadual do Ceará, Fortaleza, Ceará.

Sampaio, J. J. C. \& Santos, A. W. G. (2016). A experiência dos Centros de Atenção psicossocial e o movimento brasileiro de reforma psiquiátrica. In A. M. F. Pitta (Ed.), Reabilitação psicossocial no Brasil (pp. 163-172). São Paulo, SP: Hucitec. 
Sobre os autores

Thiago Sousa Felix é psicólogo (UNIFOR) e mestrando em Psicologia pelo Programa de Pós-graduação em Psicologia (UFC). É Bolsista da FUNCAP e membro do Paralaxe. thiagosfelix2004@yahoo.com.br

Aluísio Ferreira de Lima é doutor em Psicologia Social pela PUC-SP. Professor do Departamento de Psicologia (UFC) atua na Linha Sujeito e Cultura na Sociedade Contemporânea e é coordenador do Paralaxe. aluisiolima@hotmail.com

Certificamos que todos os autores participaram suficientemente do trabalho para tornar pública sua responsabilidade pelo conteúdo. A contribuição de cada autor pode ser atribuída como se segue: Thiago Sousa Felix e Aluísio Ferreira de Lima contribuíram para a conceitualização, investigação e visualização do artigo; Thiago Sousa Felix fez a redação inicial do artigo (rascunho) e Thiago Sousa Felix e Aluísio Ferreira de Lima são os responsáveis pela redação final (revisão e edição).

Recebido em: 22/11/2016

Revisado em: 23/07/2017

Aceito em: 26/09/2017 